\title{
Detection and Classification of Vehicles on Curved Roads
}

\author{
Venugopala P. S., Ashwini B.
}

\begin{abstract}
Detection of a vehicle is a very important aspect for traffic monitoring. It is based on the concept of moving object detection. Classifying the detected object as vehicle and class of vehicle is also having application in various application domains. This paper aims at providing an application of vehicle detection and classification concept to detect vehicles along curved roads in Indian scenarios. The main purpose is to ensure safety in such roads. Gaussian mixture model and blob analysis are the methods applied for the detection of vehicles. Morphological operations are used to eliminate noise. The moving vehicles are detected and the class of the vehicle is identified.
\end{abstract}

Index Terms: Vehicle detection, Classification, Counting, Gaussian Mixture Model, Blob analysis.

\section{INTRODUCTION}

Road safety is an important topic in the current situation where the number of vehicles increases at a rapid rate. The advancement of technology has led to the usage of automated systems for traffic surveillance. This includes detection, identification, classification, counting, tracking etc. This paper focuses on one such application.

This project is applicable on curved roads where the vision is blocked. The input video given to this project is obtained from a stationery camera installed to record the arrival of vehicles from one direction. The vehicles on the other side are notified whenever a vehicle is detected. By doing this safety is ensured.

Among the many methods available for detection of moving objects, methods like background subtraction, frame differencing, optical flow are the major ones. The Gaussian mixture model approach of background subtraction method is used in this work. Vehicles in a video sequence can be detected using foreground detector based on Gaussian mixture models. Further, the detected vehicle is classified as heavy or light based on the maximum area of bounding box surrounding it.

The remaining part of the paper describes the related works done in this area, methodology of the project,

Revised Manuscript Received on July 08, 2019.

Venugopala P. S, Associate Professor, Dept of CSE, NMAM Institute of Technology, Nitte (Affiliated to V.T.U., Belagavi).

Ashwini B, Associate Professor, Dept of ISE, NMAM Institute of Technology, Nitte (Affiliated to V.T.U., Belagavi). experimental results. Based on these results obtained conclusions are drawn.

\section{RELATED WORK}

Ashwini B. et al. [1] carried out vehicle detection, extraction of features and identification based on the detected futures. Vehicle detection is carried out using Gaussian Mixture Model. Blob analysis is used to draw the bounding boxes around the detected vehicles. Identification of detected vehicles is done on the basis of the extracted feature vectors. Maximally Stable Extremal Regions is the feature extraction algorithm used.

Prem Kumar Bhaskar et al. [2] addressed the issue of detecting vehicle and traffic data from video frames by making use of video data captured from a stationery traffic camera and estimates the number of vehicles present in a region. The tracking of vehicles is carried out only inside a particular region called count box, to avoid redundancy and provide high performance. The average accuracy obtained in this experiment is $91 \%$.

Mahesh C. Pawaskar et al. [5] proposed the background subtraction object tracking algorithm. The performance evaluation is done by experimenting with different settings of adjustable parameters. Some of those are Processing time, Recall (Detection Rate), Precision, Figure of Merit. The results have been produced for several image sequences. In this work, three different sequences are used. They are Sequence Hall Monitor, Sequence Water Surface and Sequence Moving Curtains. In this project, convolution operation is applied to the binary image to produce fast result due to parallel architecture of embedded system. The results produced using this method have good accuracy when compared to other methods.

Gottipati. Srinivas Babu [6] has proposed moving object detection and tracking based on foreground detection. Threshold values are used to identify the moving objects. Unmanned Aerial Vehicle based automatic traffic estimation applications are focused. In this algorithm, after selecting a proper threshold value each and every frame is extracted to form a histogram for background detection. Using the threshold value and histogram, the background is subtracted from the sequential frames. After detecting the moving objects, shadow removal process is carried out to calculate its area 
bounded by the vehicle. Then morphological operations are applied and bounding boxes are drawn on the moving objects. This algorithm is used for online (real time) and offline (quasi real time) video processing and it has a low computational complexity.

Barkha Malkaniya et al. [11] discussed an approach to identify the existence of moving objects in the frames of a video and keep track of them. It is a survey of various object detection and tracking methods.

\section{METHODOLOGY}

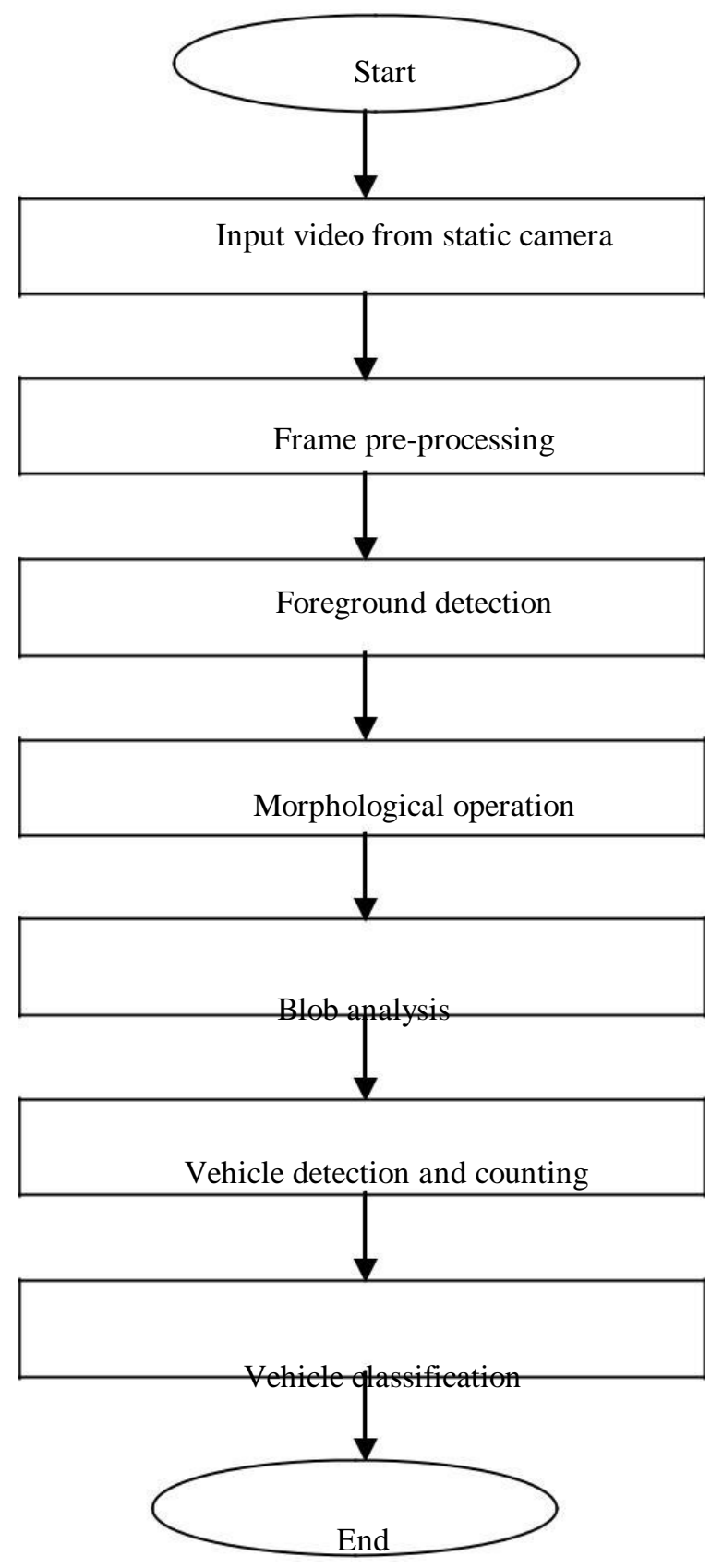

Fig 1: Flow chart for proposed approach

The method proposed in this paper obtains video from a static camera placed near a curved road. The following operations are then carried out on that video.

\section{A. Gaussian Mixture Model}

The entire video is not processed immediately. Instead an initial video frame is obtained in which the moving objects are segmented from the background. The first 25 frames are used to initialize the Gaussian mixture model by the foreground detector. The Foreground Detector System compares a background model to the video file under consideration to determine whether individual pixels are part of the background or the foreground. The model then computes a foreground mask. By using background subtraction, foreground objects in an image taken from a stationary camera can be detected.

\section{B. Morphological Operations}

Morphological operations can be used to remove noise within the object region or from the background of the image. Here we have used morphological opening to remove the undesirable noises present after foreground segmentation and to fill the gaps in detected objects.

\section{Blob analysis}

This method is used to identify the moving objects in a frame. Bounding boxes are then drawn around the detected objects. Because this method identifies every object moving in the foreground, we filter the ones that have less than 6000 pixels. This ensures that movements in leaves and far away objects are not detected. But, some such movements exceed 6000 pixels. It was observed that such small movements do not continue for more than 5 frames. Therefore, we consider only those blobs that continue for more than 5 frames as vehicles.

\section{Classification}

Detected vehicles are then classified as heavy (bus, truck) or light (car, bike) vehicles. For this we make use of the maximum area of the bounding box for a particular vehicle. The area of bounding box for that vehicle is found for every frame in which it gets detected. The maximum of these values is chosen. Heavy vehicles and light vehicles have different range of values for this property. This range cannot be generalized for all scenarios as it depends on the camera position. But it remains constant

\section{E. Technique used}

The video is processed frame by frame. In each frame the foreground is detected and 
noise removal is carried out. The bounding boxes with area less than 6000 pixels are ignored. Also, because this algorithm is applicable in narrow roads, we assume that only one vehicle passes at a time. Therefore, if

there is more than one bounding box, it has to be around smaller moving objects that are not vehicles and can be ignored. Hence, we can make an assumption that there can be only one bounding box per frame.

$[\mathrm{m}, \mathrm{n}] \beta$ [rows in bbox matrix, columns in bbox matrix] if $\mathrm{m}>=2$ then

Consider first row of bbox matrix end if

To make sure that the bounding box is around the same object, we take the difference of its $\mathrm{X}$ coordinates in the current and previous frame. This difference will be less than 50 pixels for the same object. We can ascertain that the detected object is indeed a vehicle by checking if the bounding box around that object continues for at least 5 frames.

$\mathrm{xDiff} \beta \mathrm{X}$ coordinate of bbox in current frame $-\mathrm{X}$ coordinate of bbox in previous frame if $\mathrm{xDiff}<50$ then increment number of frames in which bounding box exists

end if

If the difference happens to be more than 200, then the object detected is a new one.

if $x$ Diff $>200$ AND bbox has continued for atleast 5 frames then

it is a new vehicle

end if

After 5 frames the bounding box area is noted until the vehicle reaches minimum $X$ coordinate value if the road takes a right turn and maximum $X$ coordinate value if the road takes a left turn. When this point is reached, we get the maximum bounding box area. Based on this, the vehicles are classified as heavy or light. Also, at this point the count of vehicles detected is incremented.

if $\mathrm{X}$ coordinate equals $\mathrm{X}$ minimum (or $\mathrm{X}$ maximum) then increment count

note maximum bounding box area classify as heavy or light

end if

\section{EXPERIMENTAL RESULTS}

This section gives the overall picture of the results obtained. The data for the experiment in the form of video is obtained in curved roads. The camera is placed so as to record the front view of the approaching vehicles. Video was taken in 4 different places.

Fig 2 shows one of the scenarios where the video was taken. The road here takes a turn further. The objective is to notify the vehicles coming from above about the vehicles coming from the other side as the vision here is blocked.
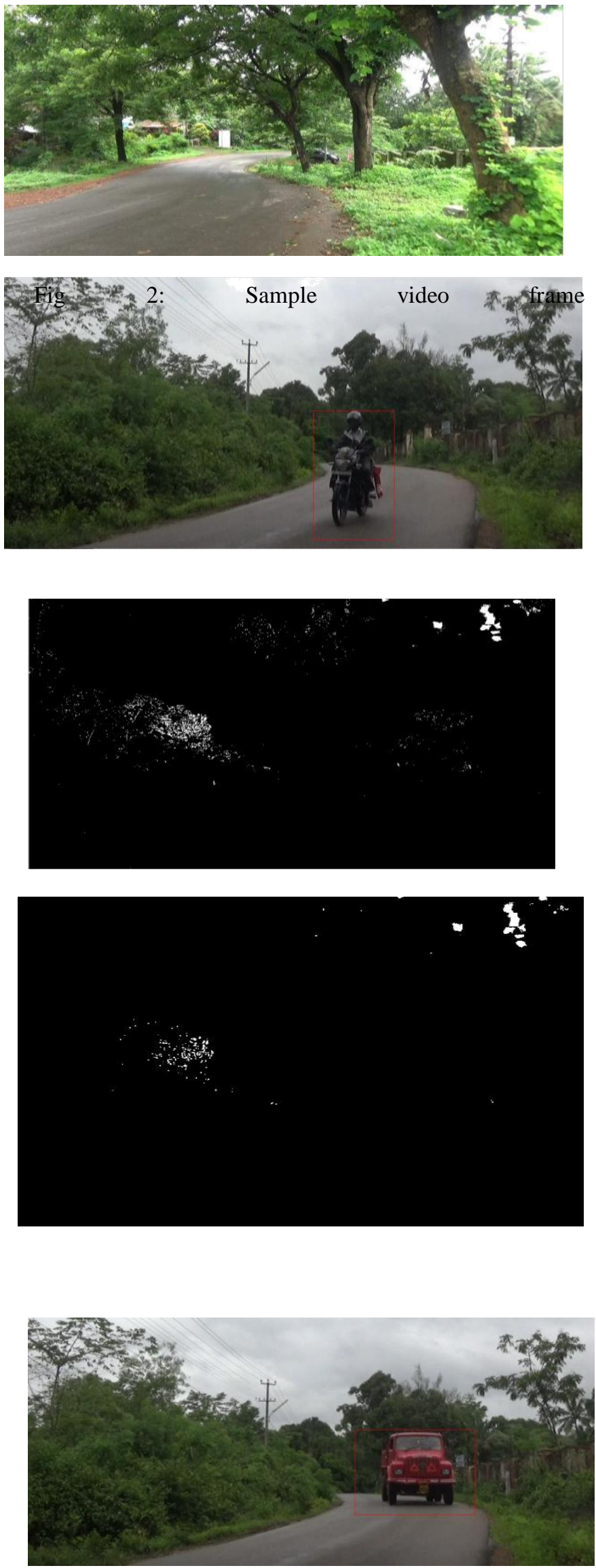

Published By:

Blue Eyes Intelligence Engineering 


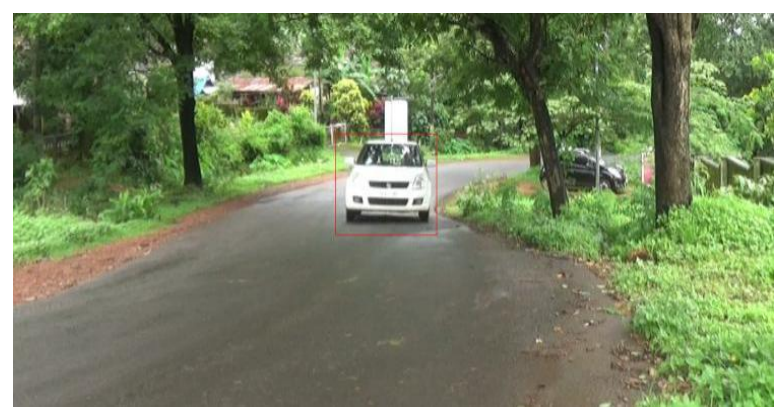

Fig 6a: Light vehicle
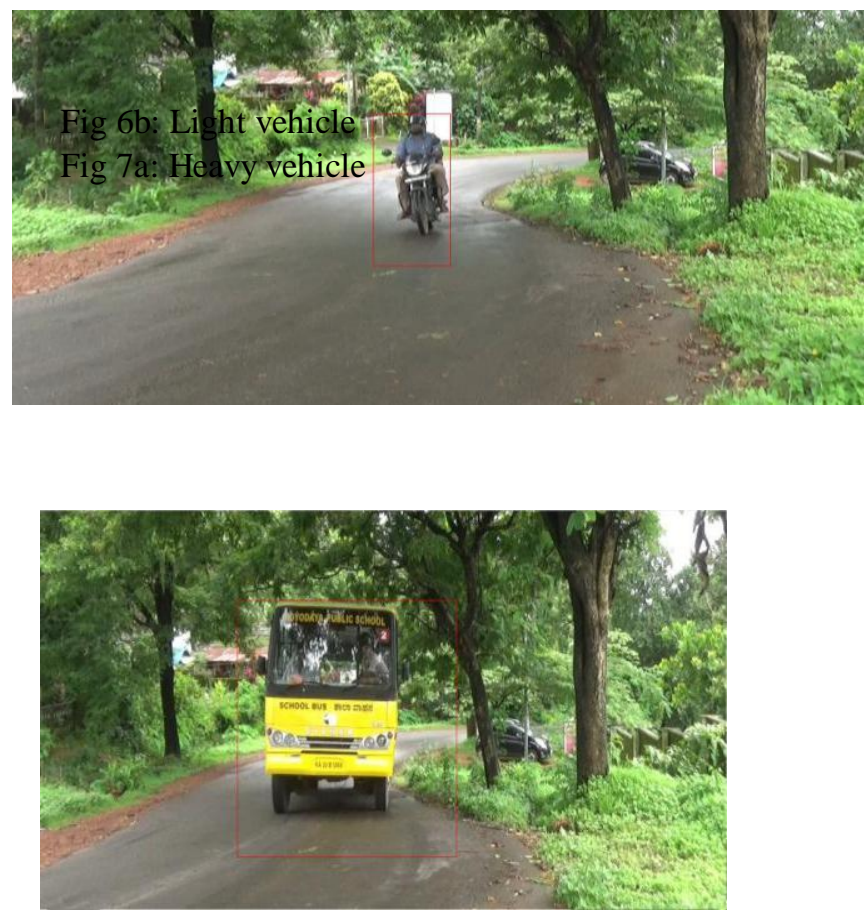

Fig 7b: Heavy vehicle

Video was also obtained in a rainy atmosphere to check efficiency. Fig $8 \mathrm{a}, 8 \mathrm{~b}$ shows detection of vehicles in such a condition. It was observed that some of the vehicles were detected a little late.

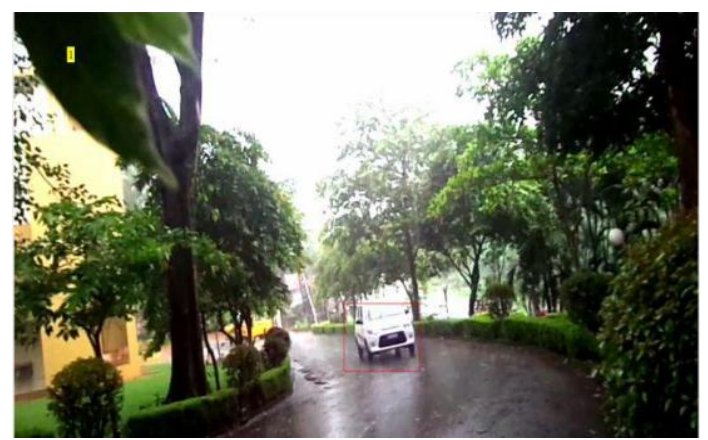

Fig 8a: Detected vehicle

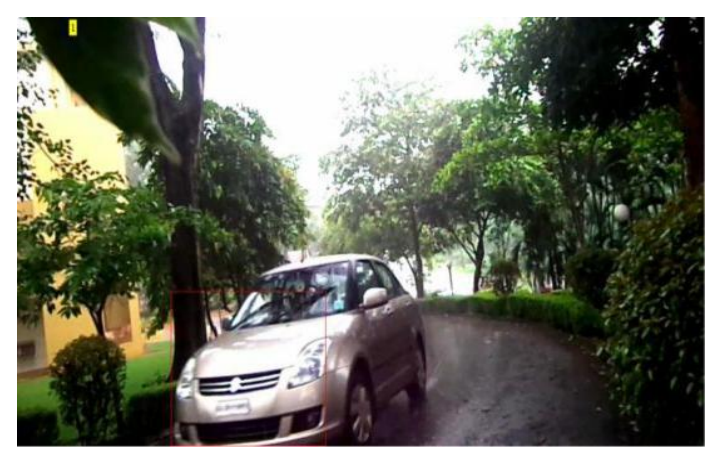

Fig 8b: Late detection

The range of maximum bounding box area for heavy or light vehicles is determined by observing the same for a few vehicles at a particular place. Though it is not the same everywhere, the range remains constant for a given place after installing the camera. Because of the large difference in range for heavy and light vehicles, they can be easily classified.

TABLE I. OBSERVATIONS

\begin{tabular}{|c|c|c|l|l|}
\hline \multirow{2}{*}{ eo Vid } & \multicolumn{2}{|l|}{ Light vehicles } & \multicolumn{2}{l|}{ Heavy vehicles } \\
\cline { 2 - 5 } & Actual & Detected & Actual & Detected \\
\hline $\begin{array}{c}\text { vid } \\
\text { eo1 }\end{array}$ & 5 & 5 & 2 & 2 \\
\hline $\begin{array}{c}\text { vid } \\
\text { eo2 }\end{array}$ & 8 & 9 & 3 & 2 \\
\hline $\begin{array}{c}\text { vid } \\
\text { e03 }\end{array}$ & 14 & 13 & 0 & 1 \\
\hline $\begin{array}{c}\text { vid } \\
\text { eo4 }\end{array}$ & 4 & 4 & 1 & 1 \\
\hline
\end{tabular}

The above table depicts the observations made in videos taken at different places. It shows the number of vehicles classified as heavy or light. We can see that the detection is efficient but there are a few errors in classification. The video 'video4' was taken in rainy weather. In this case, some of the vehicles were detected a little late due to poor lighting and contrast.

This method also counts the number of vehicles present in the video. We have observed that this method efficiently gives the count in all the video samples

\section{CONCLUSION}

In this method, moving vehicles are detected based on background subtraction method. The moving vehicles which are detected are then classified as heavy or light based on the maximum area of bounding box surrounding them. The method is simple and detects vehicles quite efficiently. It provides reliability and 
applicability in video surveillance aspects. Based on whether the road takes a right or a left turn few changes have to be made. The technique has a few limitations too. Occluded vehicles are not detected. The range of heavy and light vehicles are dependent on the road and position of the camera and hence can be decided only after observing a few vehicles through the camera.

\section{REFERENCES}

1. Ashwini B., Deepashree B., Dr. Yuvaraju B.N., Venugopala P.S., Identification of Vehicles in Traffic Video", International conference on Signal Processing, Communication, Power and Embedded System(SCOPES)-2016, DOI 10.1109/SCOPES.2016.7955507.

2. Prem Kumar Bhaskar, Suet-Peng Yong, "Image Processing Based Vehicle Detection and Tracking Method", Computer and Information Sciences(ICCOINS), 2014, DOI 10.1109/ICCOINS.2014.6868357.

3. Meenakshi Gupta, Laxmidhar Behera, Venkatesh K. Subramanian, Mo M. Jamshidi, "A Robust Visual Human Detection Approach With UKF-Based Motion Tracking for a Mobile Robot", IEEE SYSTEMS JOURNAL, Vol 9, No. 4, pp. 1363-1375, 2015.

4. Ms. Jyoti J. Jadhav, "Moving Object Detection and Tracking for Video Survelliance", International Journal of Engineering Research and General Science Volume 2, Issue 4, June-July, 2014, ISSN 2091-2730.

5. Mr. Mahesh C. Pawaskar, Mr. N. S. Narkhede, Mr. Saurabh S. Athalye, "Detection of Moving Object Based On Background Subtraction", International Journal of Emerging Trends \& Technology in Computer Science(IJETICS), Volume 3, Issue 3, May-June 2014.

6. Gottipati. Srinivas Babu, "MOVING OBJECT DETECTION USING MATLAB”, International Journal of Engineering Research \& Technology(IJERT), ISSN: 2278-0181, Vol. 1 Issue 6, August-2012.

7. Anaswara S Mohan, Resmi R, "Video Image Processing for Moving Object Detection and Segmentation using Background Subtraction",

2014 First International Conference on Computational Systems and Communications(ICCSC)| 17-18 December 2014| Trivandrum.

8. Hyeok Jang, In-Su Won, Dong-Seok Jeong, “Automatic Vehicle Detection and Counting Algorithm", IJCSNS International Journal of

Computer Science and Network Security, VOL. 14 No.9, September 2014.

9. S. R. Balaji, S. Karthikeyan, "A survey on moving object tracking using image processing", Intelligent Systems and Control(ISCO), 2017, IEEE, DOI: 10.1109/ISCO.2017.7856037.

10. Susmita A. Meshram, A.V.Malviya, "Traffic Surveillance by Counting and Classification of Vehicles from Video using Image Processing", International Journal of Advanced Research in Computer Science and Management Studies, Volume 1, Isssue 6, November 2013, ISSN: 2321-7782.

11. Barkha Malkaniya, Rupinder Kaur, Ms. Shrusti, “A Review Analysis on Real Time Moving Object Detection and Object Tracking Using Surveillance Systems", International Journal of Innovative Computer Science \& Engineering, Volume 2 Issue 3;July-August-2015; Page No. $21-24$

\section{AUTHORS PROFILE}

Venugopala P.S. Associate Professor, Dept of CSE,

NMAM Institute of Technology, Nitte (Affiliated to V.T.U., Belagavi).

Ashwini B Associate Professor, Dept of ISE, NMAM Institute of Technology, Nitte (Affiliated to V.T.U., Belagavi). 